\title{
Multi-Level Visualization of Interrelated Data Entities
}

\author{
Sandra Gama \\ INESC-ID and Instituto Superior Técnico, \\ Universidade de Lisboa \\ Av. Rovisco Pais, 1 \\ Lisboa, Portugal \\ sandra.gama@tecnico.ulisboa.pt
}

\author{
Daniel Gonçalves \\ INESC-ID and Instituto Superior Técnico, \\ Universidade de Lisboa \\ Av. Rovisco Pais, 1 \\ Lisboa, Portugal \\ daniel.goncalves@inesc-id.pt
}

\begin{abstract}
Nowadays, electronic devices are part of our daily routines, resulting in information generation at virtually any time and context. Due to different styles of interaction, data produced by human activities is not only in considerable quantities, but it is also extremely rich, which makes it difficult to manage and analyze. Visualization has the potential to overcome this limitation: not only is it an excellent means to display large quantities of information, but it also alleviates cognitive load associated with data interpretation. We created an interactive multi-level layered visualization, in which time may be represented sequentially through layers. Data entities are displayed as circles with size proportional to a particular data feature we need to highlight, allowing immediate comparison between entities. By selecting an entity, we may see, through visual connectors, all the interrelated entities over the different time layers. User tests have shown that our visualization makes important information immediately perceivable, in a way that is easy to navigate and analyze.
\end{abstract}

\section{Categories and Subject Descriptors}

H.5.2 [Information interfaces and presentation]: User Interfaces

\section{General Terms \\ Human Factors}

\section{INTRODUCTION}

Human activities, with increasing use of electronic devices, generate high quantities of information in a variety of contexts. Such amounts of information are often quite complex and difficult to interrelate and analyze. Visualization, as an excellent means to display large quantities of data alleviates cognitive load associated with information interpretation [2], may be the solution to this shortcoming. We created a visualization that displays data items in a timely manner and allows interactive exploration, providing mechanisms for in- terrelating and comparing items. It is minimalistic and easy to navigate, allowing an effective analysis. We adopted a multi-level layered view, in which each level corresponds to a time frame, where data items are depicted. This visualization makes general information immediately perceivable and allows interactive manipulation for further exploration of patterns such as relationships among entities and detailed information on a given entity.

In section 2 we present our visualization and in section 3 we discuss user evaluation results, drawing several conclusions from our study.

\section{APPROACH}

We created an interactive visualization, consisting of a main area and several additional elements, as depicted in Figure 1. The main area is composed of a multi-level interactive layered visualization, in which each level corresponds to a time frame. Data entities are displayed as circles with size proportional to a particular feature that it verifies. We provide the means to represent the positive and negative aspects of a particular feature, or both: the entity circle is sub-divided into two semicircles displaying information both on positive and negative aspects through western conventional positivenegative color coding [2]. The leftmost, green, semicircle represents the positive aspect and the rightmost, red, semicircle, displays the negative aspect of the feature. Semicircle size is proportional to the magnitude of both the positive and the negative aspects. This mechanism makes important information instantly perceivable. In fact, it makes it possible to promptly understand the entities for which there are only positive (or negative) aspects to a given feature and also allows the immediate comparison between entities, without requiring further data exploration.

Moving the mouse over a circle highlights it by assigning more saturation to its present color hue, making it more noticeable, and displays information on entity relationships. The current entity is linked to all the entities with which it has any type of interrelation through visual line connectors. Line connector thickness is proportional to the interrelation strength between two entities (which may be defined as a specific feature such as entity similarity): the thicker the line, the stronger the relationship is. Color is assigned to each interconnection individually, in order to avoid visual confusion and allowing immediate line discrimination. We did not use fully saturated colors, in order to keep our visual artifacts from competing for the user's visual attention [2]. 


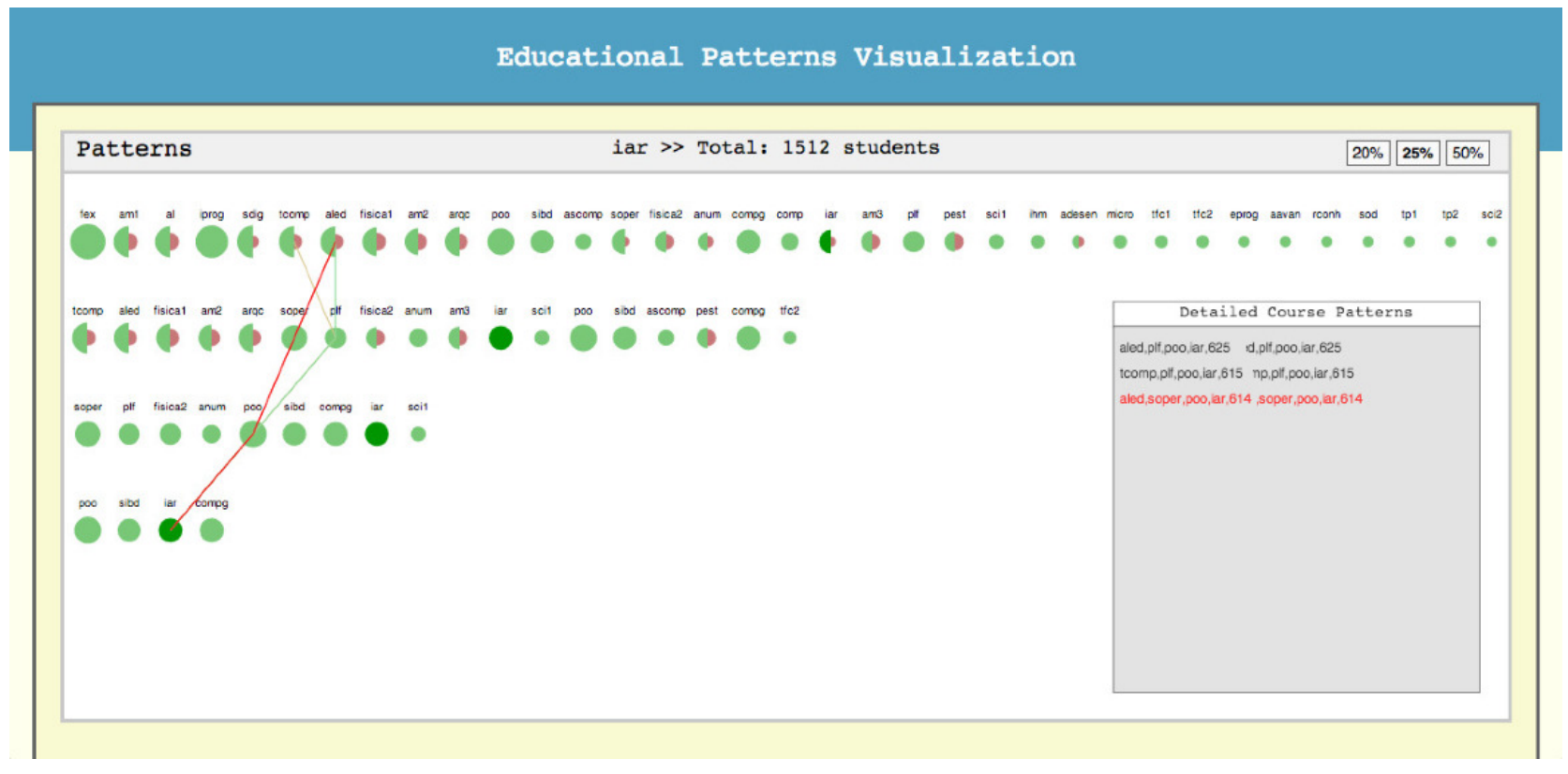

Figure 1: A visualization for educational data mining patterns.

When the mouse leaves the entity circle, this information is no longer displayed. Furthermore, whenever an entity is selected, additional information is displayed at the top of the visualization, and information on entity interconnection is displayed on the detailed entity interconnections' panel. The total number of relationships is shown at the upmost part of the panel and each interconnection is listed below, as displayed in Figure 1. If there is a high number of interrelations for the currently selected entity, a scroll bar is automatically shown.

Our visualization allows the interactive comparison of different entities. As previously mentioned, moving the mouse over a specific entity circle displays information on the interrelations with other entities on the main visualization area. However, if we select one data entity (by clicking it), it locks the currently selected entity and displays its information throughout the visualization without clearing it when the mouse leaves the selected circle. Moving the mouse to any other entity will also display its information, making it possible to compare entities and their interrelations over time, as depicted in Figure 1.

There are three main aspects to our visualization. The first is immediate information reading. In particular, we can immediately perceive the number time frames represented, as well as the number of entities for each time frame. Additionally, we can immediately recognize entity features depicted by size. In Figure 1 we can see that four time frames are depicted, as well as the courses for each level and the positive and negative aspects of its represented data feature are immediately perceived by both green and red radiuses. Another important aspect is data exploration. Our visualization shows more information regarding a particular entity whenever it is selected and allows the comparison between two different entities.

\section{EVALUATION}

We conducted a study with 10 users, who were given a verbal introduction to the study, and handed a list of questions to answer, corresponding to particular tasks. Time and number of errors were measured and a satisfaction questionnaire with two parts was then performed: the SUS (System Usability Scale) [1] in conjunction with a small set of questions to evaluate information gathering. Evaluation yielded very promising results. Users were able to obtain information for task completion, while time and error rates showed that the system scales well for large amounts of information and that users easily learned how to use it. Finally, user satisfaction questionnaires proved our system's usability to be very good.

\section{CONCLUSIONS}

Large amounts of data emerge from different human activities, which are difficult to manage and interrelate. We created a visualization which combines different interaction mechanisms, revolving over a layered representation of time frames. User tests have shown that our visualization made general information immediately available, such as the number of represented time frames and the entities with more positive or negative aspects of a particular feature. It provides the means to display large amounts of information in a way which provides the means for analysis and makes general information explicit that was not evident otherwise.

\section{ACKNOWLEDGMENTS}

This work was supported by national funds through FCT Fundação para a Ciência e a Tecnologia, under project Educare - PTDC/EIA-EIA/110058/2009 and INESC-ID multiannual funding - PEst-OE/EEI/LA0021/2013.

\section{REFERENCES}

[1] J. Brooke. Sus: A quick and dirty usability scale, 1996.

[2] C. Ware. Information Visualization: Perception for Design. Elsevier, 2012. 